\title{
ANTECIPAÇÃO DE SAFRA PARA VIDEIRA NIÁGARA ROSADA NA REGIÃO SUL DO ESTADO DE MINAS GERAIS ${ }^{1}$
}

\author{
Harvest anticipation for ‘Niagara Rosada’ grapes in southern Minas Gerais, Brazil
}

\author{
Ester Alice Ferreira ${ }^{2}$, Murillo de Albuquerque Regina ${ }^{3}$, \\ Nilton Nagib Jorge Chalfun ${ }^{4}$, Luís Eduardo Corrêa Antunes ${ }^{3}$
}

\section{RESUMO}

Este experimento foi conduzido em propriedade particular no município de Caldas-MG, durante o ciclo 1999/ 2000, utilizando plantas pré-estabelecidas da cultivar Niagara Rosada enxertadas sobre RR 101-14, visando a antecipar o período de colheita da videira no sul do Estado de Minas Gerais. Foi comparado o efeito da poda antecipada, do uso de cobertura plástica e da irrigação com o sistema de produção, por meio dos seguintes tratamentos: poda antecipada, com cobertura plástica, com e sem irrigação; poda antecipada e poda convencional. Avaliaram-se o comprimento do ciclo, acúmulo de graus-dia da poda à maturação, características físicas: diâmetro transversal e longitudinal, peso e número de cachos por planta e as características químicas: teor de sólidos solúveis totais, $\mathrm{pH}$ e acidez total titulável. Apesar do maior acúmulo de graus-dia, a cobertura plástica não promoveu redução no ciclo vegetativo e causou redução na produção. A poda antecipada sem cobertura plástica permitiu uma precocidade de 42 dias na colheita, sem aumentar o custo de produção do vinhedo. As plantas submetidas à poda convencional a céu aberto apresentaram maior número e peso de cachos, maiores valores de diâmetro e teor de SST. Não houve diferença significativa entre pH e acidez.

Termos para indexação: Graus-dia, Vitis sp., cobertura plástica.

\begin{abstract}
This work was carried out in a private vineyard, in Caldas-MG, during the 1999/2000 agricultural year, using 'Niagara Rosada' grafted on 'RR 101-44' established plants, aiming to anticipate grape harvest in Southern Minas Gerais, Brazil. The following treatments were evaluated: anticipated pruning with plastic cover, with or without irrigation; anticipated and regular pruning. Cycle length, degrees-day accumulation from pruning to ripening, morphological characteristics (transversal and longitudinal diameters, weight and number of bunches per plant) and chemical characteristics (soluble solids level, $\mathrm{pH}$ and acidity) were evaluated. Despite the higher degree-day accumulation, the plastic cover did not promote a reduction in the vegetative cycle and reduced the production. The anticipated pruning without plastic cover allowed precocity of 42 days in the harvest without any increase in the costs. Plants submitted to regular pruning, without any cover, presented higher number and weight of bunches, higher diameter and soluble solids level. There were no significant differences among the treatments with regard to $\mathrm{pH}$ and acidity of the fruits.
\end{abstract}

Index terms: Degrees-days, Vitis sp., plastic cover.

(Recebido para publicação em 8 de outubro de 2003 e aprovado em 8 de julho de 2004)

\section{INTRODUÇÃO}

A videira 'Niágara Rosada’ destaca-se como uma das uvas de mesa preferidas pelo consumidor brasileiro, sendo, atualmente, a principal cultivar plantada nos Estados de São Paulo, Santa Catarina, Minas Gerais (TERRA, 1996; POMMER et al., 1997) e está também entre as uvas de mesa mais comercializadas nos principais entrepostos do País (Central de Abastecimento de Minas Gerais, 1990; Agrianual, 2000).

Devido à sazonalidade climática, sua produção é restrita a alguns períodos do ano. Na região do sul de Minas, sua colheita concentra-se nos meses de ja- neiro a fevereiro, coincidindo com a produção de Jundiaí - SP, o que provoca uma queda acentuada nos preços.

Nesse contexto, o produtor que conseguir antecipar sua safra obterá maiores lucros. Para isso, o uso de práticas que visem a alterar o ciclo buscando a antecipação do período de colheita seria uma opção para aumentar o período de oferta.

Os estudos envolvendo a relação entre o comprimento do ciclo e a temperatura do ar mostram que em regiões onde a temperatura é mais elevada, o ciclo da cultura é menor, em razão de seu crescimento acelerado

1. Extraído da Dissertação de Mestrado da primeira autora. Universidade Federal de Lavras/UFLA - Caixa Postal 3037 - $37200-000$ - Lavras, MG Programa de Pós-graduação em Agronomia/Fitotecnia.

2. Engenheira Agrônoma, Msc., Doutoranda - Departamento de Agricultura/UFLA, ester@ufla.br

3. Engenheiro Agrônomo, PhD., Pesquisador da EPAMIG - Fazenda Experimental de Caldas, Caixa Postal 33 - $37780-000$ - Caldas, MG. Fone: (35) 3735-1101, murillo@epamigcaldas.gov.br

4. Engenheiro Agrônomo, Dr., Professor Titular do Departamento de Agricultura da UFLA - Fone: (35) 3829-1339. 
(SENTELHAS, 1998). Segundo Pedro Júnior et al. (1993), a cv. Niágara Rosada completa o seu ciclo mediante temperaturas superiores a $10^{\circ} \mathrm{C}$, e, baseado no conceito de graus-dia (GD), a necessidade térmica dessa cultivar para se desenvolver, da poda à colheita, é de 1549 graus-dia, independente da época de poda.

Para se obter um aumento na temperatura, o cultivo protegido com cobertura plástica tem sido uma das alternativas mais usadas por viticultores de outros países, permitindo a manipulação do ciclo e, conseqüentemente, a antecipação no período de oferta. (FAO, 1990; CIRAMI e FURKALIEV, 1991; ANTONACCI, 1993; SOUSA, 1996).

No Brasil, essa técnica foi testada com sucesso no Rio Grande do Sul por Shiedeck et al. (1997), apresentando como resultado o aumento da temperatura no interior da estufa de plástico, e tendo como principal efeito a redução no comprimento do ciclo vegetativo da videira, em várias épocas de poda e independente dessas.

A necessidade de validar um sistema de produção, visando à antecipação de safra na região do sul do Estado de Minas Gerais, levou à condução do presente trabalho, com o objetivo de verificar o efeito da poda antecipada e da cobertura plástica no comprimento do ciclo e as alterações no comportamento da cv. Niagara Rosada.

\section{MATERIAL E MÉTODOS}

O experimento foi realizado durante o ciclo 1999/2000 em vinhedo de propriedade particular no município de Caldas/MG, situado a $1150 \mathrm{~m}$ de altitude, $21^{\circ} \mathrm{S}$ e $40^{\circ} \mathrm{W}$, cujo clima apresenta temperatura média anual de $19^{\circ} \mathrm{C}$ com médias mínimas de $13^{\circ} \mathrm{C}$ e máximas de $26^{\circ} \mathrm{C}$, umidade relativa de $75 \%$ e precipitação pluviométrica de $1500 \mathrm{~mm}$ anuais.

Foram utilizadas videiras da cv. Niagara Rosada com 11 anos de idade, enxertadas no porta-enxerto RR 101-14, apresentando produtividade média de 6 kg/ planta, conduzidas em espaldeira, com espaçamento de 2,0 m entre plantas e 1,60 m entre linhas.

O delineamento experimental foi inteiramente casualizado, com três repetições, seis plantas por parcela, considerando-se parcela útil as quatro plantas centrais. Os tratamentos foram: T1- poda antecipada com cobertura plástica e irrigação; T2- poda antecipada com cobertura sem irrigação; T3- poda antencipada e T4poda convencional, sem aplicação de Dormex ${ }^{\circledR}$.

A poda antecipada foi realizada em 21 de julho de 1999 e imediatamente após, foi aplicado Dormex®, $5 \%$, com pulverizador costal. A poda convencional foi realizada em 27 de agosto de 1999.

A estrutura utilizada para suporte da cobertura plástica foi construída em cada parcela, de forma a se adaptar às condições do vinhedo já instalado e foi disposta no sentido das linhas de cultivo. O modelo desenvolvido foi construído de madeira, no qual passavam fios de arame que davam sustentação ao plástico, que foi do tipo filme de polietileno difusor - DTR Nortene ${ } \quad$ (Figura 1).

O sistema de irrigação foi o de aspersão localizada na subcopa, utilizando os equipamentos já instalados na propriedade, o qual se iniciou dois dias após a poda, em 23/07. A vazão do aspersor utilizado foi de 8 L/min, irrigando cada planta por 1hora três vezes por semana até 05/09.

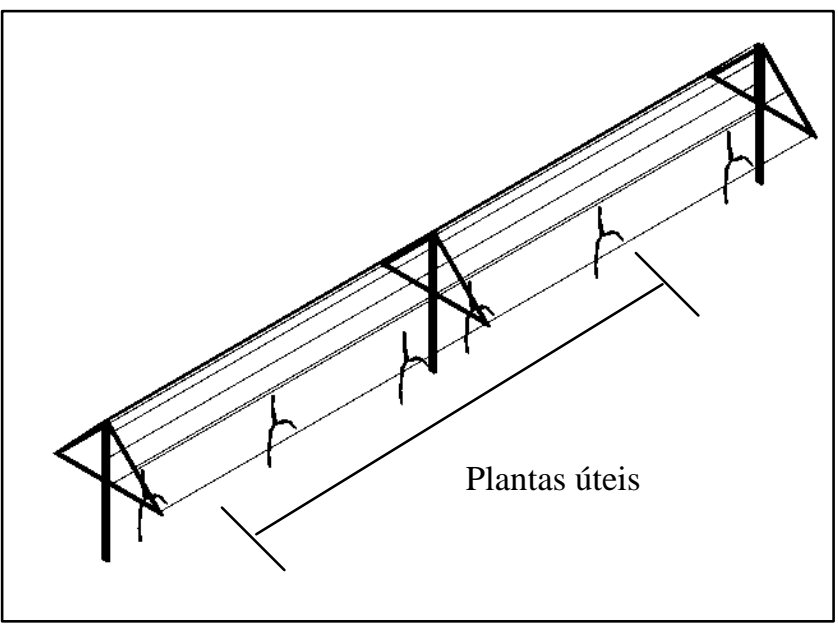

FIGURA 1 - Esquema das estruturas utilizadas como suporte para cobertura plástica. Caldas, MG. 
Para acompanhamento da temperatura e umidade, foram instalados dois termohigrógrafos: no interior do cultivo protegido e em ambiente externo, sem cobertura plástica. Foram registrados os valores diários de temperatura máxima e mínima do ar, coletados semanalmente.

Utilizou-se a equação de Winkler (1948), citado por Hidalgo (1993), para calcular a soma de graus-dia, dentro e fora da estufa, adotando como temperatura base $10^{\circ} \mathrm{C}: \mathrm{GD}=\Sigma\left(\mathrm{Tm}-10^{\circ} \mathrm{C}\right) \times \mathrm{n}^{\mathrm{o}}$ dias da poda à colheita; em que: Tm- Temperatura média diária.

Foram avaliados o início e o fim das principais fases que caracterizam o ciclo fenológico de desenvolvimento da videira, de acordo com a metodologia proposta por Carbonneau (1981), a saber: Brotação- início quando a planta apresentou no mínimo dois esporões diferentes e fim quando a porcentagem de gemas nessa fase atingiu 50\%. Floração - inicio com duas flores abertas em duas inflorescencias distintas e fim quando apenas duas inflorescências não possuíam todas as flores abertas e; Maturação - que se baseou na mudança na cor das bagas (pintor), tendo início quando apenas dois cachos distintos apresentaram mudança na cor das bagas e fim quando apenas dois cachos não apresentaram todas as bagas coloridas.

Ao final do subperíodo fenológico maturação, quando mais de $50 \%$ das bagas apresentavam a coloração característica da cultivar 'Niagara Rosada', foram realizadas inspeções semanais e o ponto de colheita foi determinado visualmente, utilizando os critérios adotados pelo produtor.

A colheita, então, foi realizada em 22 de dezembro de 1999 para os tratamentos de poda antecipada (21/07/99) e em 4 de fevereiro de 2000 para as plantas submetidas à poda convencional (27/08/99). Na ocasião da colheita, foram avaliados o número e o peso dos cachos por planta.

Uma amostragem aleatória foi realizada retirando-se 20 cachos por tratamento. Esses foram macerados em sacos plásticos para obtenção do mosto e análises de pH, SST e acidez total.

Os dados foram analisados pelo Programa SISVAR versão 3.04 (FERREIRA, 1998) e comparados pelo teste de médias de Scott e Knott.

\section{RESULTADOS E DISCUSSÃO}

No Quadro 1 verificam-se o somatório de grausdia para os diferentes tratamentos e a respectiva duração do ciclo.
QUADRO 1 - Somatório de Graus-dia e comprimento do ciclo para os diferentes tratamentos.

\begin{tabular}{|c|c|c|}
\hline Tratamentos & Graus - dia & $\begin{array}{c}\text { Comprimento } \\
\text { do ciclo }\end{array}$ \\
\hline $1^{* *}$ & 1424,6 & 154 dias \\
2 & 1424,6 & 154 dias \\
3 & 969,6 & 154 dias \\
4 & 1324,1 & 158 dias \\
\hline
\end{tabular}

Foi observado que, embora as temperaturas registradas em ambiente protegido tenham sido superiores às de céu aberto, houve semelhança entre os Tratamentos 1, 2 e 4 quanto aos valores de Graus-dia. Essa semelhança pode ser atribuída ao fato de as plantas cultivadas a céu aberto terem se desenvolvido quando as temperaturas estavam mais elevadas, propiciando um comportamento similar ao das plantas cultivadas em cobertura plástica.

Por outro lado, o desenvolvimento das plantas submetidas somente à poda antecipada e céu aberto (Tratamento 3) ocorreu em condições de temperatura inferior aos demais tratamentos (fim de inverno e início de primavera), sendo esse fato responsável pelo baixo acúmulo de graus-dia.

No Quadro 2, é apresentada a duração média em dias dos subperíodos fenológicos.

Pelos resultados encontrados, constata-se que não houve diferença significativa entre os tratamentos para os diferentes subperíodos fenológicos. Resultados semelhantes foram registrados por Shiedeck (1996) em videiras com varas envoltas por tubos de polietileno de baixa densidade, que não apresentaram antecipação na brotação nem variação no comprimento total do ciclo.

Maiores diâmetros transversal e longitudinal, maior número de cachos e a maior produção foram obtidos nas plantas cultivadas a céu aberto (Quadro 3).

Tal fato pode estar associado à ocorrência de desavinho decorrente do não-vingamento dos frutos (SOUSA, 1996). Resultados semelhantes quanto à baixa produtividade sob cultivo protegido, quando comparadas a céu aberto, foram verificados por Vrynoides (1976) e Antonacci (1993), que observaram redução no peso de bagas de videira sob cultivo protegido na ordem de $11 \%$.

Os valores médios das características químicas para os diferentes tratamentos são aprentados no Quadro 4. 
QUADRO 2 - Valores médios da duração (dias) dos subperíodos fenológicos da videira Niagara Rosada para os tratamentos, nos diferentes subperíodos fenológicos: Início e Fim de Brotação (IB) e (FB), Floração (IF e FF) e Maturação (IM) e (FM).

\begin{tabular}{|c|c|c|c|c|c|c|}
\hline Tratamentos & IB & FB & IF & FF & IM & FM \\
\hline T1** & $15 \mathrm{~ns}$ & $25 \mathrm{~ns}$ & $50 \mathrm{~ns}$ & $58 \mathrm{~ns}$ & $131 \mathrm{~ns}$ & $147 \mathrm{~ns}$ \\
$\mathrm{~T} 2$ & $16 \mathrm{~ns}$ & $27 \mathrm{~ns}$ & $51 \mathrm{~ns}$ & $58 \mathrm{~ns}$ & $130 \mathrm{~ns}$ & $151 \mathrm{~ns}$ \\
$\mathrm{~T} 3$ & $15 \mathrm{~ns}$ & $23 \mathrm{~ns}$ & $52 \mathrm{~ns}$ & $58 \mathrm{~ns}$ & $130 \mathrm{~ns}$ & $147 \mathrm{~ns}$ \\
$\mathrm{~T} 4$ & $21 \mathrm{~ns}$ & $29 \mathrm{~ns}$ & $54 \mathrm{~ns}$ & $63 \mathrm{~ns}$ & $134 \mathrm{~ns}$ & $153 \mathrm{~ns}$ \\
\hline
\end{tabular}

**Tratamento 1 - Poda Convencional ( Realizada em 27 de agosto de 1999)

Tratamento 2 - Poda antecipada + cobertura

Tratamento 3 - Poda antecipada + irrigação + cobertura plástica

Tratamento 4 - Poda antecipada (Realizada em 21 de julho de 1999)

QUADRO 3 - Valores médios das características físicas para os diferentes tratamentos.

\begin{tabular}{|c|c|c|c|c|}
\hline Tratamentos & Número de cachos/planta & $\begin{array}{c}\text { Produção } \\
\text { (kg/planta) }\end{array}$ & $\begin{array}{c}\text { Diâmetro transver- } \\
\text { sal (cm) }\end{array}$ & $\begin{array}{c}\text { Diâmetro Longitudinal } \\
\text { (cm) }\end{array}$ \\
\hline $1^{* *}$ & $9,66 \mathrm{~b} *$ & $1,76 \mathrm{~b}$ & $1,8 \mathrm{~b}$ & $1,7 \mathrm{~b}$ \\
2 & $8,00 \mathrm{~b}$ & $1,50 \mathrm{~b}$ & $1,9 \mathrm{~b}$ & $1,6 \mathrm{~b}$ \\
3 & $19,66 \mathrm{a}$ & $3,96 \mathrm{a}$ & $2.2 \mathrm{a}$ & $1,8 \mathrm{a}$ \\
4 & $23,00 \mathrm{a}$ & $5,01 \mathrm{a}$ & $2,2 \mathrm{a}$ & $1,9 \mathrm{a}$ \\
\hline
\end{tabular}

QUADRO 4 - Valores médios das características químicas para os diferentes tratamentos.

\begin{tabular}{|c|c|c|c|}
\hline Tratamentos & $\mathbf{p H}$ & Teor de Sólidos Solúveis Totais & Acidez (meq/L) \\
\hline $1^{* *}$ & $3,096 \mathrm{a}^{*}$ & $11,60 \mathrm{~b}$ & $100,65 \mathrm{a}$ \\
2 & $3,146 \mathrm{a}$ & $12,11 \mathrm{~b}$ & $80,93 \mathrm{a}$ \\
3 & $3,176 \mathrm{a}$ & $13,37 \mathrm{a}$ & $80,58 \mathrm{a}$ \\
4 & $3,116 \mathrm{a}$ & $13,79 \mathrm{a}$ & $70,19 \mathrm{a}$ \\
\hline
\end{tabular}

*Valores seguidos de mesma letra, em linhas diferentes, não diferem entre si pelo teste de Scott e Knott

**Tratamento 1 - Poda Convencional ( Realizada em 27 de agosto de 1999)

Tratamento 2 - Poda antecipada + cobertura

Tratamento 3 - Poda antecipada + irrigação + cobertura plástica

Tratamento 4 - Poda antecipada (Realizada em 21 de julho de 1999)

Observaram-se menores teores de Sólidos Solúveis Totais para os tratamentos com cobertura plástica. Esse resultado difere do verificado por Vrynoides (1976), Manzo e Ruggieri (1988) e Antonacci (1993) que não registraram diferenças significativas quanto aos valores de SST entre as plantas conduzidas sob cultivo protegido e a céu aberto. Já Shiedeck (1996) relata resultados positivos do uso da cobertura plástica, que promoveu nas plantas sob cultivo protegido teores de sólidos solúvies totais mais acentuados do que nas cultivadas a céu aberto.

Como a cobertura plástica foi retirada antes que se iniciasse a maturação dos frutos, o que ocorreu neste ensaio pode ser explicado baseando-se nas considerações de Fernandez (1991). Segundo o referido autor, o acúmulo de açúcares nas bagas, dentro dos limites de

Ciênc. agrotec., Lavras, v. 28, n. 6, p. 1221-1227, nov./dez., 2004 
cada cultivar, está relacionado com o tamanho delas, sendo imprescindível que essa aumente em volume, para que haja maior acúmulo de açúcares. Baseado nos dados de diâmetro longitudinal e transversal apresentados anteriormente (Quadro 2), é possível associar os baixos teores de ${ }^{\circ} \mathrm{Brix}$ ao tamanho reduzido das bagas dos Tratamentos 1 e 2.

É importante ressaltar que o desavinho e a heterogeneidade da maturação das bagas nos Tratamentos 1 e 2 podem ter afetado os ${ }^{\circ}$ Brix pela mistura de bagas em diferentes estágios de maturação na ocasião da análise.

Os resultados das análises de acidez total e real do mosto $(\mathrm{pH})$ diferiram dos encontrados por Manzo e Rugierri (1988), Antonacci (1993) e Shiedeck (1996), que verificaram, para ambas variáveis, superioridade das plantas submetidas ao cultivo protegido em relação a céu aberto. O que ocorreu no presente ensaio pode ser explicado baseando-se nas considerações de Fernandez (1991), que atribui a diminuição da acidez durante o processo da maturação a dois fatores: a diluição em função da grande quantidade de água que é absorvida durante maturação e a diminuição dos ácidos málico e tartárico durante a respiração dos frutos. Pela ação da diluição, pode-se explicar a semelhança entre os tratamentos, tanto para acidez quanto para $\mathrm{pH}$, que tiveram o processo de maturação de suas bagas coincidente com um período de chuvas abundantes na região.

Na Figura 2, observam-se as diferenças entre os tratamentos e também a melhor visualização da superioridade do tratamento 4 em relação aos demais tratamentos.

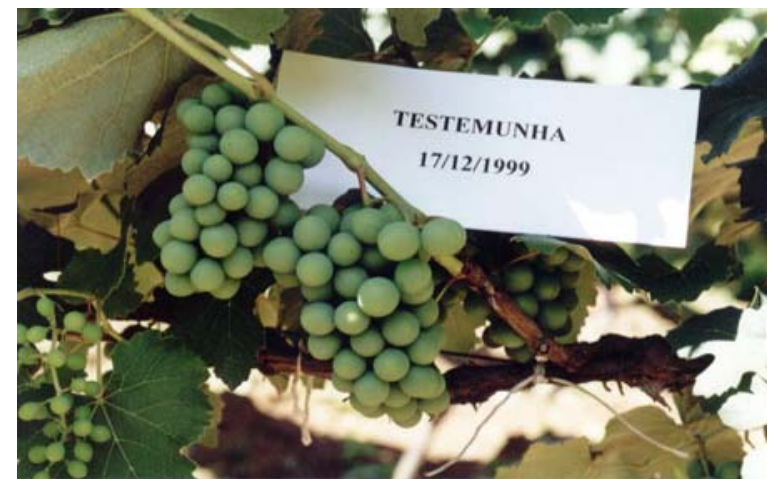

TRATAMENTO 1

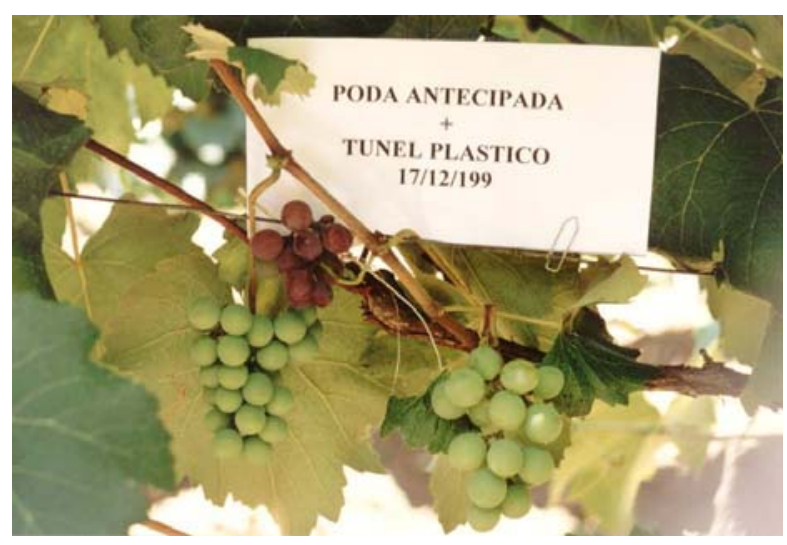

TRATAMENTO 3

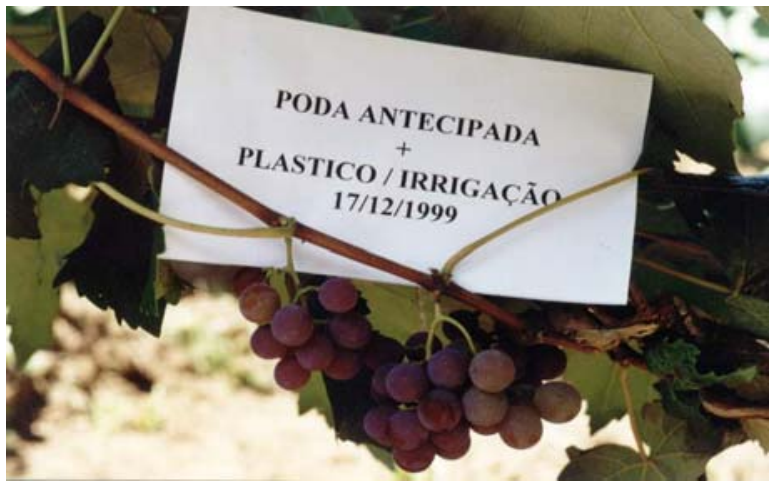

TRATAMENTO 2

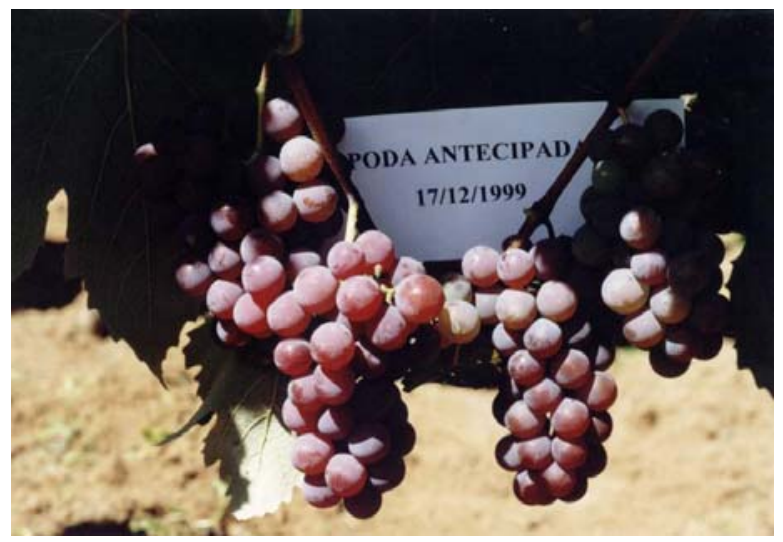

TRATAMENTO 4

FIGURA 2 - Comparação dos diferentes tratamentos aplicados na cultivar Niágara Rosada, visando à antecipação de safra na ocasião da colheita. 
É importante ressaltar o fato de que a antecipação de 42 dias obtida com o uso da poda antecipada Tratamento 4, ocorreu às vésperas das festas de fim ano, ocasião em que o mercado encontra-se aquecido pela grande procura por uva, sendo esse resultado promissor aos produtores da região.

A crescente valorização da cv. Niágara Rosada estimula sua produção. Tendo em vista a colheita coincidente de algumas regiões e ainda a possibilidade de produção da ' Niágara Rosada' em regiões tropicais, fazem-se necessárias mais pesquisas na área de manipulação da colheita, para dar respaldo aos produtores da região do sul de Minas. O uso da cobertura plástica pode ser considerado uma alternativa em novos ensaios, atentando para o tipo de plástico e/ou estrutura de suporte e tempo de cobertura.

\section{CONCLUSÕES}

a) A antecipação da poda permitiu 42 dias de precocidade da colheita, com uma produção de 11,88 t/ha.

b) A cobertura plástica, apesar de promover maior acúmulo de graus-dia, não permitiu a antecipação da colheita.

c) O uso da cobertura plástica induziu à ocorrência de desavinho das flores e conseqüente redução na produção por planta.

d) Não houve diferença significativa entre $\mathrm{pH}$ a acidez total titulável e as plantas cultivadas a céu aberto apresentaram maiores teores de sólidos solúveis totais.

\section{AGRADECIMENTO}

À Empresa de Pesquisa Agropecuária de Minas Gerais EPAMIG - Fazenda Experimental de Caldas, pelo apoio na condução deste ensaio.

\section{REFERÊNCIAS BIBLIOGRÁFICAS}

AGRIANUAL 2000. São Paulo: Agros, 2001. 435 p.

ANTONACCI, D. Comportamento produtivo di nove cultivar di uve da tavola coltivata in ambiente protetto. Vignevini, Roma, v. 20, n. 1/2, p. 53-62, 1993.

CARBONNEAU, A. Observation sur vigne: codification des donnes 'es agronomiques. Viti, Bourdeaux, v. 5, n. 2, p. 9-13, août 1981.
CENTRAL DE ABASTECIMENTO DE MINAS GERAIS. Acompanhamento da oferta e preço médio de produtos Ceasa: unidade $\mathrm{BH}$ : comercialização de uva cultivar Niagara Rosada de 1/1990 à 12/1995: classificação alfabética. 1990. Disponível em: <http:// www.agridata.gov.mg.br/>. Acesso em: 15 abr. 2000.

CIRAMI, R. M.; FURKALIEV, D. G. Effect of time of pruning and hidrogen cyanamide on grwoun Cardinal grapes. Australian Journal of Experimental Agriculture, Muriootpa, v. 31, n. 2, p. 273-278, 1991.

FERNANDEZ, M. de T. Biologia de la vid: fundamentos biológicos de la viticultura. Madrid: Mundi, 1991. $349 \mathrm{p}$.

FERREIRA, F. D. SISVAR: sistema de análise de variância para dados balanceados. Lavras: DCE/UFLA, 1998. 18 p. Programa em disquete.

FOOD AND AGRICULTURE ORGANIZATION OF THE UNITED NATION. Plant prodution in protection paper: protected cultivation in mediterranean climat. Rome, 1990. 313 p.

HIDALGO, L. Tratado de viticultura. Madrid: Mundi, 1993. $983 \mathrm{p}$.

MANZO, P.; RUGGIERI, L. M. Premières experiences de semi-forçage du raisin de table recoce "Matilde". Plasticulture, Paris, v. 1, n. 77, p. 4-10, 1988.

PEDRO JÚNIOR, M. J. et al. Caracterização fenológica da videira 'Niágara Rosada' em diferentes regiões paulistas. Bragantia, Campinas, v. 52, n. 2, p. 153-160, 1993.

POMMER, C. V. et al. Variedades de videira para o Estado de São Paulo. Campinas: IAC, 1997. 53 p. (Boletim técnico, 166).

SCHIEDECK, G. Ecofisiologia da videira e qualidade da uva 'Niágara rosada' conduzida sob estufa de plástico. 1996. 111 f. Dissertação (Mestrado em Fitotecnia) - Universidade Federal do Rio Grande do Sul, Porto Alegre, 1996.

SCHIEDECK, G. et al. Fenologia da videira 'Niagara Rosada' cultivada em estufa e a céu aberto. Revista Brasileira de Agrometeorologia, Santa Maria, v. 5, n. 2, p. 199-206, 1997. 
SENTELHAS, P. C. Aspectos climáticos para viticultura tropical. Informe Agropecuário, Belo Horizonte, v. 19, n. 194, p. 9-14, 1998.

SOUSA, J. S. I. de. Uvas para o Brasil. Piracicaba: FEALQ, 1996. 791 p.

TERRA, M. M. A produção de uvas no Sul do Estado de Minas Gerais. In: ENCONTRO SUL MINEIRO DE
FRUTICULTURA DE CLIMA TEMPERADO, 1., 1996, Poços de Caldas. Anais... Poços de Caldas: [s.n.], 1996. p. $12-22$.

VRYNOIDES, P. Novelles acquisitions en matière de culture de raisins de table: culture du Cardinal sois matière plastique. Bulletin de l'O.I.V., Limassol, v. 49, n. 550, p. 965-978, 1976. 